\title{
Early cardiac changes following anthracycline chemotherapy in breast cancer: a prospective multi-centre study using advanced cardiac imaging and biochemical markers
}

\author{
Suchi Grover ${ }^{1,3^{*}}$, Carmine DePasquale ${ }^{1,3}$, Darryl P Leong ${ }^{1}$, Adhiraj Chakrabarty ${ }^{3}$, Kerry A Cheong ${ }^{4}$, Dusan Kotasek ${ }^{4}$, \\ Rohit Joshi ${ }^{5}$, Amy Penhall', Lucas Jeorg ${ }^{1}$, Majo Joseph ${ }^{1,3}$, Bogda Koczwara ${ }^{2,3}$, Joseph Selvanayagam ${ }^{1,3}$
}

From 15th Annual SCMR Scientific Sessions

Orlando, FL, USA. 2-5 February 2012

\section{Summary}

This prospective study is designed to identify novel imaging (utilizing cardiovascular magnetic resonance and advance echocardiography) and biochemical markers to detect early, sub-clinical cardiotoxicity following chemotherapy.

\section{Background}

Cardiac toxicity is an important long term side effect of anthracyline chemotherapy. We hypothesized that novel cardiovascular magnetic resonance (CMR) and echocardiographic markers of myocardial function, oedema, and necrosis can detect early, subclinical cardiac toxicity in patients receiving anthracycline therapy for breast cancer.

\section{Methods}

29 chemonaive patients with breast cancer (early stage and metastatic), underwent serial cardiovascular magnetic resonance imaging (for LV volumes/ejection fraction, myocardial oedema and necrosis), advanced echocardiography (for LV strain in addition to standard measurements of LV diastolic function), pro brain natriuretic peptide (pro-BNP) and high-sensitivity Troponin $\mathrm{T}$ (hs-TnT, Roche assay) measurements. All tests were conducted at baseline, 1 month, and 3 months following commencement of anthracyclines. 6 normal volunteers ( 3 male, 3 female) underwent the same CMR protocol. For oedema imaging, a triple inversion

${ }^{1}$ Cardiology, Flinders Medical Centre, Adelaide, SA, Australia

Full list of author information is available at the end of the article recovery fast-spin echo sequence (short-TI inversion recovery) was used in 3 short-axis views of the left ventricle. For assessment of myocardial oedema, the ratio of mean signal intensity (SI) of the myocardium was compared to that of skeletal muscle.

\section{Results}

In the study patients, the mean CMR LV end-systolic volume index (LVESVI) increased from baseline of 17.8 \pm 6.2 to $20.3 \pm 5.9 \mathrm{~mL} / \mathrm{m} 2(\mathrm{p}<0.001)$ at 1 month and remained significantly elevated at 3 months $(22.7 \pm 5.6$ $\mathrm{mL} / \mathrm{m} 2 ; \mathrm{p}<0.001)$. Similarly, the end-diastolic volume index (LVEDVI) increased from $62.9 \pm 11.6 \mathrm{~mL} / \mathrm{m} 2$ at 1 month to $66.4 \pm 11.8(\mathrm{p}<0.000)$ at 3 months. Echocardiographic measurement of global longitudinal strain (GLS) showed no significant change at 1 month, however decreased in absolute magnitude from $-21.4 \pm 3 \%$ baseline to $-19.5 \pm 1 \%(\mathrm{p} \leq 0.001)$ at 3 months. Left ventricular diastolic function did not change significantly in study patients, either 1 or 3 month post chemotherapy. BNP did not correlate significantly with observed changes in LVEDV, LVESV and GLS. Hs-TnT did not change at 1 month but increased from $3.8 \pm 1.6$ to 10.7 \pm 9.8 at 3 months $(\mathrm{p} \leq 0.003)$. Although there was no difference between baseline values of $\mathrm{T} 2$ ratio between normal volunteers and study patients, $15 / 26$ (58\%) of patients had an abnormal T2 signal (SI increase to $>1.9$ as specified by Lake Louis criteria) in one or more short axis slices post chemotherapy. There was no new late gadolinium hyperenhancement to suggest focal myocardial necrosis/fibrosis in any patient following therapy.

(C) 2012 Grover et al; licensee BioMed Central Ltd. This is an open access article distributed under the terms of the Creative Commons 


\section{Conclusions}

Our data suggest that there are subtle functional changes in the LV myocardium early post anthracycline chemotherapy that can be detected by both CMR and advanced echocardiographic techniques. This is likely mediated by myocardial inflammation (without necrosis). These findings may be a basis for development of early predictors of cardiac damage and earlier intervention/preventative strategies.

\section{Funding}

This study has received a grant from FMC Foundation, Adelaide, Australia.

\section{Author details}

${ }^{1}$ Cardiology, Flinders Medical Centre, Adelaide, SA, Australia. ${ }^{2}$ Oncology,

Flinders Medical Centre, Adelaide, SA, Australia. ${ }^{3}$ Flinders University, Adelaide, SA, Australia. ${ }^{4}$ Adelaide Cancer Centre, Adelaide, SA, Australia. ${ }^{5}$ Lyell McEwin

Hospital, Adelaide, SA, Australia.

Published: 1 February 2012

- Convenient online submission

- Thorough peer review

- No space constraints or color figure charges

- Immediate publication on acceptance

- Inclusion in PubMed, CAS, Scopus and Google Scholar

- Research which is freely available for redistribution 